\title{
PROPAGACÃO DAS ONDAS DE ROSSBY NOS INVERNOS DE MÁXIMA FREQÜÊNCIA DE OCORRÊNCIA DE GEADAS NA PAMPA ÚMIDA
}

\author{
GABRIELA V. MÜLLER ${ }^{1}$, SIMONE E T. FERRAZ² E TÉRCIO AMBRIZZI ${ }^{3}$ \\ ${ }^{1}$ Centro de Investigaciones Científicas y Transferencia de Tecnología a la Producción, \\ Diamante (CICYTTP-CONICET), Entre Ríos, Argentina \\ ${ }^{2}$ Departamento de Física, Universidade Federal de Santa Maria RS, Brasil \\ ${ }^{3}$ Departamento de Ciências Atmosféricas, Instituto de Astronomia, \\ Geofísica e Ciências Atmosféricas, Universidade de São Paulo SP, Brasil
}

gabrielamuller@cicyttp.org.ar, simonefe@smail.ufsm.br, ambrizzi@model.iag.usp.br

Recebido Novembro 2007 - Aceito Dezembro 2008

\begin{abstract}
RESUMO
Neste trabalho estudou-se a influência dos padrões de onda extratropicais, que favorecem o desenvolvimento de eventos extremos frios no sudeste Sul-Americano, e em particular na região conhecida como Pampa Úmida. O aquecimento anômalo observado na região do oceano Pacífico tropical ocidental a nordeste da Austrália, durante os invernos de máxima freqüência de ocorrência de Geadas Generalizadas (GG) no centro-leste da Argentina, (região conhecida como Pampa Úmida - PU), atua como disparador de ondas de Rossby, as quais se propagam até o continente, favorecendo assim a ocorrência daqueles eventos. O padrão de propagação obtido nas simulações numéricas com um modelo baroclínico global, mostra o predomínio de um número de onda 3. Adicionalmente, foram analisadas as correlações do vento meridional em altos e baixos níveis observados para os eventos de GG, selecionados dentro dos invernos de máxima freqüência de ocorrência desses eventos. O vento meridional global em $250 \mathrm{hPa}$ apresenta regiões com correlação estatisticamente significativa com o vento meridional médio na PU. A configuração obtida no caso do vento meridional global em $250 \mathrm{hPa}$, correlacionado com o vento meridional na PU, pode estar associada ao padrão de propagação das ondas simuladas numericamente a partir da forçante tropical. Igualmente importantes e significativos são os valores de correlação do vento sul nos baixos níveis, em particular para toda região da PU. O padrão de ondas simulado está bem representado pelas significativas correlações entre o vento meridional hemisférico em altos níveis e a temperatura no dia de evento de GG.
\end{abstract}

Palavras-chaves: ondas de Rossby, geadas, conveccão, Pampa Úmida

ABSTRACT: ROSSBY WAVE PROPAGATION DURING WINTERS OF MAXIMUM FREQUENCY OF FROST OCCURRENCE OVER THE WET PAMPA

The influence of extratropical waves on the development of extreme cold events over southeast South America, particularly on the Argentinean Wet Pampa region is studied in this work. An anomalous heating observed over the occidental tropical Pacific Ocean, northeast of Australia, during Austral winters of maximum frequency of Generalized Frost (GF) occurrence over the center-east of Argentina, known as Wet Pampa (WP), acts as a Rossby wave source which generate waves that propagate towards the South American continent favoring the frost events. The wave propagation pattern obtained from simulations using a Global Baroclinic Model shows wavenumber 3 dominance. Additional, upper and lower levels meridional wind correlations during the GF events selected were analyzed. The $250 \mathrm{hPa}$ global meridional wind shows a significant correlation (0.9) with the meridional wind at the WP region. The wave propagation pattern observed in this case agrees with that simulated by the model when a heating source is located at the Pacific tropical ocean. Also, significant correlation values were found for the low level southern winds at the WP region. The wave pattern simulated shows a good correlation between the hemispheric meridional wind at higher levels and the air temperature in the day of GF events.

Keywords: Rossby waves, Frost, Convection, Argentinean Wet Pampa 


\section{INTRODUÇÃO}

Como pode ser visto amplamente na literatura, as ondas de Rossby modificam a estrutura do fluxo em altos níveis e modulam as características dos sistemas sinóticos. As forçantes locais destas ondas podem ser fontes de convecção localizadas em latitudes tropicais (Nogués-Paegle e Mo, 1988; Berbery e Nogués-Paegle, 1993; Mo e Higgins, 1998) ou advecção de vorticidade do vento divergente (Sardeshmukh e Hoskins, 1988), que atua como fonte de energia de ondas de Rossby em regiões de forte gradiente meridional do vento.

A teoria linear de ondas de Rossby através da análise dos padrões de teleconexão proporciona uma valiosa ferramenta para compreender os mecanismos físicos envolvidos em diferentes processos, como por exemplo, baixa temperatura em uma extensa região. Este é o caso das Geadas Generalizadas (GG) no centro - leste da Argentina, região conhecida como Pampa Úmida (PU). Nesta região durante os invernos que apresentam uma maior freqüência destes eventos, existe uma maior atividade de ondas de Rossby que alcançam o continente Sul Americano (Müller et al. 2005). Nesse sentido Müller e Ambrizzi (2007) mostram um exemplo de como a grande escala condiciona os eventos extremos frios na região da PU. Os autores apresentaram resultados numéricos dos padrões de teleconexão, que provocam as GG e permitem compreender os mecanismos dinâmicos que atuam na evolução dos mesmos, além da sua origem. Os eventos de GG que ocorrem durante os invernos de máxima freqüência de ocorrência na PU estão associados a um duplo trem de ondas com origem no Oceano Índico Tropical. Este trem duplo propaga-se pelo jato subtropical e polar no Hemisfério Sul e suas fases se unem perto do continente. Como o padrão de propagação é essencialmente dependente da estrutura do estado básico e da forçante remota (Ambrizzi et al., 1995), havendo mudança nesta última, o padrão obtido poderá ser diferente.

Ondas quase estacionárias forçadas pelo aumento da atividade convectiva, resultam em dispersão de ondas de Rossby sobre a esfera (Hoskins e Karoly, 1981; Ambrizzi e Hoskins,
1997). A localização e a intensidade da forçante convectiva, junto com a estrutura do fluxo médio afetam a fase de tais ondas (Ting e Sardeshmukh, 1993).

Neste estudo, investigou-se a relação da convecção tropical observada e o padrão de propagação das ondas planetárias durante os invernos de máxima freqüência de ocorrência dos eventos de GG. Para o desenvolvimento deste trabalho utilizaou-se um Modelo Baroclinico de Circulação Geral para testes de hipóteses e correlação simples entre diferentes campos meteorológicos, usando a região da Pampa Úmida como ponto base.

\section{CRITÉRIOS, DADOS E METODOLOGIA}

\subsection{Critérios de Seleção}

Considerou-se Geada a ocorrência de temperatura mínima igual ou inferior a $0^{\circ} \mathrm{C}$, medida em abrigo meteorológico. Para sua identificação foram consideradas temperaturas mínimas de 41 estações meteorológicas do Serviço Meteorológico Nacional (SMN-Argentina) e do Instituto Nacional de Tecnologia Agropecuária (INTA-Argentina), no período de 1961-1990. A base de dados utilizada foi analisada e consistida por Müller et al. (2000), que classifica as geadas segundo um critério de extensão espacial, agrupando-as em isoladas, parciais e generalizadas. Estas últimas, em particular, se definem quando mais de $75 \%$ das estações meteorológicas tem temperatura mínima diária inferior a $0^{\circ} \mathrm{C}$ (ver detalhes em Müller, 2006).

O estado básico de máxima freqüência de ocorrência de GG é formado pelos invernos (Junho-Julho-Agosto - JJA) dos anos de 1970, 1976 e 1988 (Müller et al., 2005), e é apresentado na tabela 1 .

\subsection{Dados}

Os dados utilizados para as diferentes análises são provenientes da Reanálise do NCEP/NCAR, com resolução espacial de $2,5^{\circ}$ x $2,5^{\circ}$ (Kalnay et al., 1996). Esta versão

Tabela 1 - Eventos seleccionados de GG (adaptado de Müller et al, 2005).

\begin{tabular}{|c|l|}
\hline \multicolumn{2}{|c|}{ EVENTOS } \\
\hline 1970 & $24 / 06,07 / 07,08 / 08,25 / 08$ \\
1976 & $11 / 06,26 / 06,04 / 07,14 / 08$ \\
1988 & $01 / 06,14 / 06,24 / 06,05 / 07,24 / 07,26 / 08$ \\
\hline
\end{tabular}


dos dados foi disponibilizada pelo "NOAA-CIRES Climate Diagnostic Center" (www.cdc.noaa.gov). São utilizados dados de vento meridional em $850 \mathrm{hPa}$ e $250 \mathrm{hPa}$. No texto é utilizado o termo "vento meridional global" para os dados em todo o globo, e "vento meridional médio" para a média do "vento meridional global" na região de $27^{\circ} \mathrm{S}-40^{\circ} \mathrm{S}$ e $54^{\circ} \mathrm{W}-57^{\circ} \mathrm{W}$ (Pampa Úmida).

Além destes são usados dados de temperatura mínima de superfície na região da PU provenientes do SMN e INTA.

\subsection{Modelo}

O uso de um modelo baroclínico tem como objetivo testar a hipóteses da importância da forçante convectiva na produção dos padrões observados, quando se têm geadas generalizadas. O modelo utilizado nos experimentos numéricos é o IGCM ("Intermediate Global Circulation Model - Center for Global Atmospheric Modeling, University of Reading") sendo uma ferramenta apropriada para investigar os mecanismos físicos de propagação de ondas, pois a resposta obtida pode ser atribuída diretamente à fonte de perturbação. Este modelo foi amplamente utilizado em diversos estudos, tais como: Jin e Hoskins (1995); Ambrizzi e Hoskins (1997), Cavalcanti (2000), Marengo et al. (2002). Mesmo não incluindo os processos físicos vinculados as mudanças de fase do vapor d'água, muitos aspectos da dinâmica atmosférica podem ser reproduzidos, desde sistemas da escala sinótica (Hoskins e Simmons, 1975) até perturbações de grande escala (Valdes e Hoskins, 1989).

O IGCM usa uma representação espectral na horizontal e um esquema de diferenças finitas na vertical. No modelo original baseado em Hoskins e Simmons (1975), o esquema de diferenças finitas conservava massa e energia total, mas não momento angular. No modelo atual foi incluído um esquema vertical alternativo baseado no esquema usado no European Centre for Médium Weather Forecasting - ECMWF (Simmons e Burridge, 1981), que conserva o momento angular. No experimento realizado neste trabalho é utilizado um truncamento no número de onda 42 (T42). Esse truncamento espectral tem associado uma grade espacial equivalente a 128 longitudes (globais) e 32 latitudes gaussianas (por hemisfério). Doze camadas são usadas na vertical: $100 \mathrm{hPa}, 150 \mathrm{hPa}, 200 \mathrm{hPa}, 250 \mathrm{hPa}, 300 \mathrm{hPa}, 400 \mathrm{hPa}$, $500 \mathrm{hPa}, 600 \mathrm{hPa}, 700 \mathrm{hPa}, 850 \mathrm{hPa}, 925 \mathrm{hPa}$ e $1000 \mathrm{hPa}$. O modelo inclui uma difusão interna bi-harmônica com coeficiente $2.333 \times 10^{6} \mathrm{~m}^{-1} \mathrm{~s}^{-1}$ e o passo no tempo em todas as integrações é de 48 minutos. A difusão é necessária para suavizar gradientes muito intensos e espúrios, que eventualmente apareçam devido à truncagem do modelo.

A execução do modelo se realiza em dois passos. O primeiro consiste na criação do estado básico inicial, a partir do qual o modelo alcança o seu estado estacionário. As variáveis utilizadas são geopotencial em superfície, componente meridional e zonal do vento e temperatura, em 12 níveis de pressão.

O segundo passo consiste em perturbar o modelo com uma forçante térmica. Esta fonte de aquecimento possui uma forma elíptica apresentando um núcleo positivo, e que pode ser posicionado conforme o experimento. O perfil vertical do aquecimento no núcleo positivo apresenta máximo aquecimento em $400 \mathrm{hPa}$, com uma magnitude de $5 \mathrm{~K} /$ dia e a média na coluna é de 2,5 K/dia, que é equivalente ao calor latente associado com $1 \mathrm{~cm}$ de precipitação por dia (Hoskins e Karoly, 1981; Hoskins e Jin, 1991).

\subsection{Separação de ondas}

Foi feita uma decomposição em números de ondas, utilizando a transformada rápida de Fourier (Magaña e Yanai, 1995) para os campos de vento meridional nos invernos de máxima freqüência de ocorrência de GG. O resultado apresentado é referente ao número de onda 3 , que foi escolhido para facilitar a comparação com outros estudos (p.ex., Magaña e Ambrizzi, 2005), os quais sugerem que este seja um dos números de onda preferenciais de propagação trópicos-extratropicos na América do Sul.

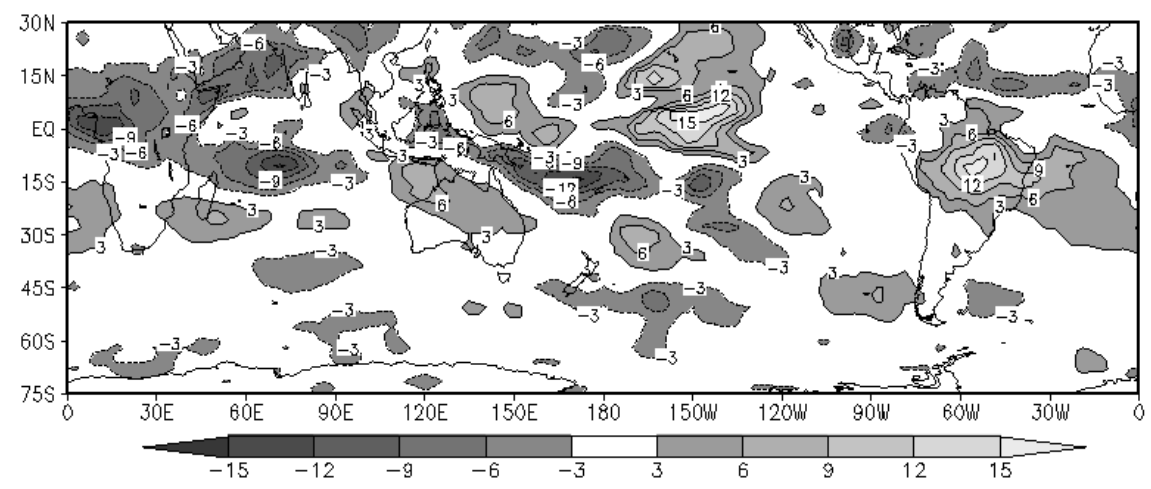

Figura 1 - Anomalias de OLR $\left(\mathrm{W} / \mathrm{m}^{-2}\right)$ nos invernos de máxima freqüência de ocorrência de GG, (Junho a Agosto: 1970, 1976 e 1988). 


\subsection{Correlações}

Como o uso de correlações é uma forma simples de relacionar duas variáveis, forem feitos os cálculos das correlações considerando como ponto base a região da $\mathrm{PU}$ $\left(27^{\circ} \mathrm{S}-40^{\circ} \mathrm{S}\right.$ e $\left.65^{\circ} \mathrm{W}-57^{\circ} \mathrm{W}\right)$. Inicialmente, foi feita uma média da temperatura mínima observada em superfície na região da PU para as datas de GG e calculada a correlação desta com vento meridional global em $250 \mathrm{hPa}$. Além disso, também foi calculada uma média do vento meridional em altos e baixos níveis nas datas de GG na mesma área e correlacionada com vento meridional global em 250 e $850 \mathrm{hPa}$. Foram considerados apenas valores estatisticamente significativos a um nível de 95\%, o teste utilizado foi T Student (Wilks, 1995).

Como a definição de geadas generalizadas é um critério espacial (Müller, 2006) e não pontual, optou-se por utilizar uma média do vento meridional obtido diretamente da Reanálise ao invés de valores de estação.

\section{RESULTADOS}

\subsection{Resultados Simulados}

Através da integração do modelo IGCM, que utilizou um o estado básico baseado na composição de eventos de máxima freqüência de ocorrência de GG, e uma forçante de calor posicionada sobre a região tropical de convecção intensa observada nas composições dos invernos selecionados, foi possível avaliar a relação trópicos-extratropicos existente nesta situação. A forçante de calor mencionada é determinada a partir das anomalias de radiação de onda longa (OLR), que resultam da composição dos invernos de máxima freqüência de ocorrência, calculados em relação ao período base de 1974-1996, e que como se observa na figura 1 , apresenta convecção anômala no Pacífico tropical em torno de $170^{\circ} \mathrm{E}-15^{\circ} \mathrm{S}$.

Na figura 2 é apresentado o campo de anomalia de vento meridional em $250 \mathrm{hPa}$, dia 13 do simulado para o estado básico

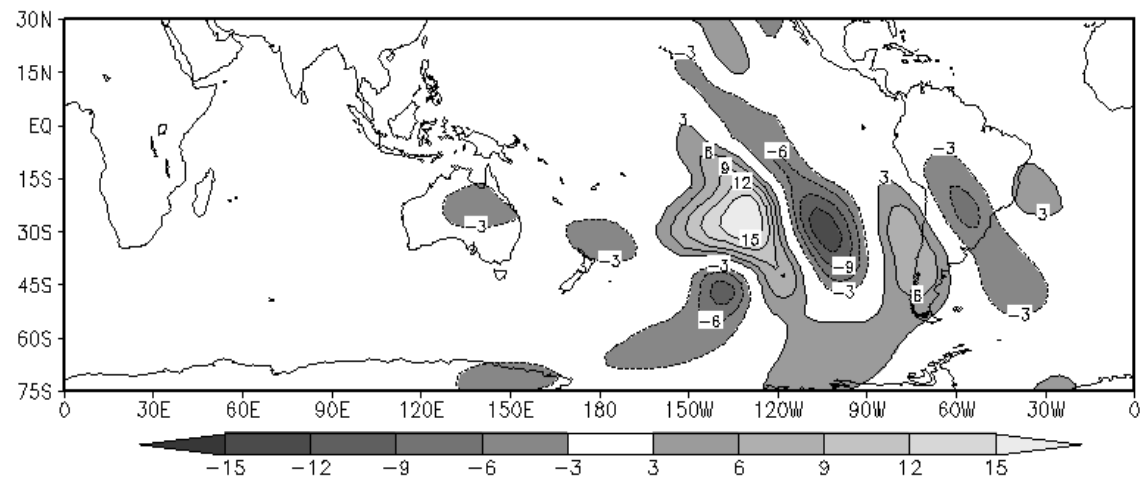

Figura 2 - Anomalias de vento meridional em $250 \mathrm{hPa}$, simulando a convecção observada em $170^{\circ} \mathrm{E}-15^{\circ} \mathrm{S}$ (local da forçante).

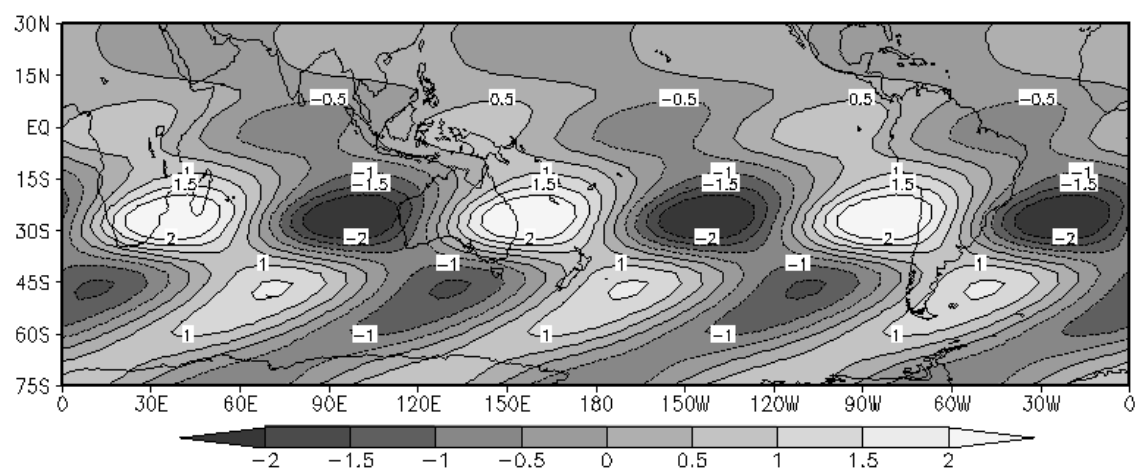

Figura 3 - Contribuição do número de onda 3 do campo de vento meridional em altos níveis. 
médio gerado através da média dos 14 eventos de GG nos invernos de máxima freqüência de ocorrência. Observa-se um padrão de propagação de ondas de Rossby, a partir da região da forçante térmica, com uma trajetória que atravessa todo o Oceano Pacifico até alcançar a América do Sul, possuindo um número de onda 3. Esse resultado é compatível com a teoria de propagação de ondas de Rossby, através do cálculo de trajetórias obtido por Müller e Ambrizzi (2006). Naquele trabalho se considerou como ponto inicial dos diferentes números de onda, a mesma região utilizada neste estudo. Os caminhos de propagação resultantes mostraram que o número de onda 3 é o único que alcança a América do Sul, o que justifica utilizar este número na separação de ondas.

A figura 3 mostra a contribuição do número de onda 3 na propagação das ondas de Rossby, apresentadas na figura 2. Pode-se notar que há boa correspondência entre os dois campos, principalmente na região de interesse, ou seja, ao sul da América do Sul, com uma anomalia de vento meridional afetando grande parte do continente. Este resultado se mostra robusto quando se analisa o comportamento dos outros números de onda (figura não mostrada) onde os padrões observados são completamente distintos. Análise similar foi realizada com a onda 3 obtida dos dados da Reanálise, onde os resultados mostraram-se compatíveis com os obtidos aqui e, portanto, não foram apresentados.

\subsection{Resultados Observados}

As figuras 4 e 6 apresentam as correlações do vento meridional médio calculado na região da $\mathrm{PU}$ em altos $(250 \mathrm{hPa}) \mathrm{e}$ baixos níveis $(850 \mathrm{hPa})$ com o vento meridional em todo o globo para os mesmos níveis, enquanto a figura 5 mostra a correlação do vento meridional em $850 \mathrm{hPa}$ com a temperatura média em superfície. Foram considerados os dias apresentados na tabela 1 e mostrado apenas os casos sem defasagem no tempo.

Observa-se na figura 4 um padrão de onda que se estende por todo o Pacífico, com valores de correlação que alcançam um máximo de 0.9 nas regiões de interesse. Além dos altos valores sobre a região da $\mathrm{PU}$, sendo esperado em função da correlação global desta variável com ela mesma, são encontrados valores significativos de correlação sobre o Pacifico, em regiões próximas as apresentadas na figura 2 . Analisando em detalhes o padrão apresentado na figura 4 em relação a figura 2, nota-se que ambos possuem características bem similares. Este resultado sugere que este padrão de propagação das ondas parece ser preferencial para gerarem os eventos de GG.

Análise similar à apresentada acima é mostrado na figura 5 , que apresenta a correlação entre o vento meridional em altos níveis em todo o globo e a temperatura na região da PU. Neste caso, valores significativos são observados apenas a partir de

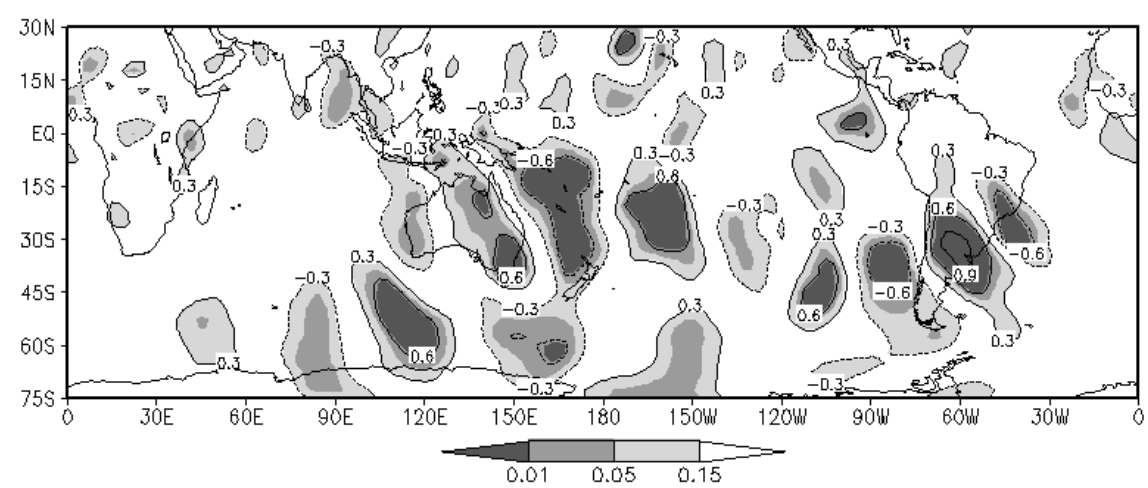

Figura 4 - Correlação entre o vento meridional em $250 \mathrm{hPa}$ e o vento meridional médio em $250 \mathrm{hPa}$ na região de $27^{\circ} \mathrm{S}-40^{\circ} \mathrm{S}$ e $54^{\circ} \mathrm{W}-57^{\circ} \mathrm{W}(\mathrm{Pampa}$ Úmida).

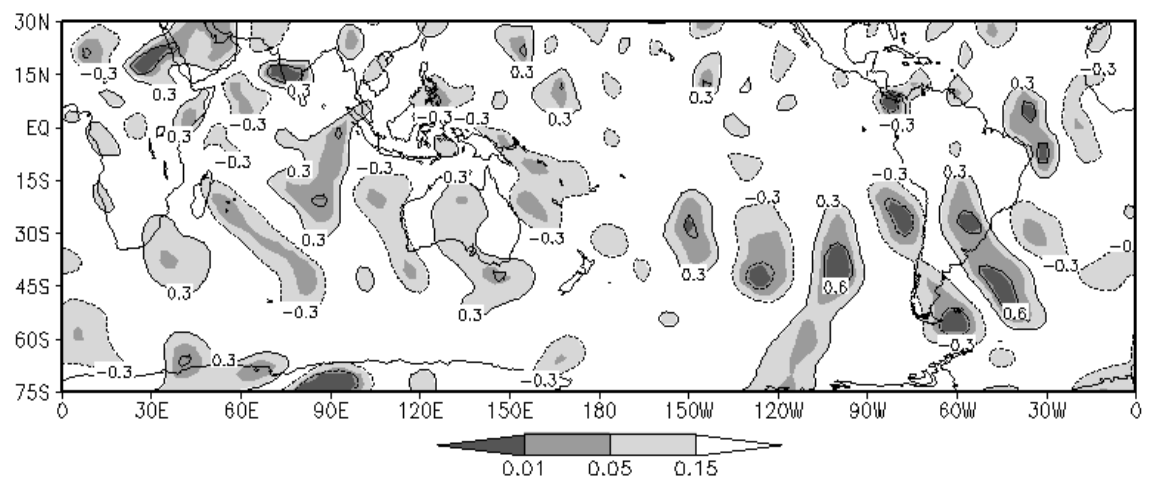

Figura 5 - Correlação entre o vento meridional em $250 \mathrm{hPa}$ e a temperatura em superfície na região de $27^{\circ} \mathrm{S}-40^{\circ} \mathrm{S}$ e $54^{\circ} \mathrm{W}-57^{\circ} \mathrm{W}(\mathrm{Pampa}$ Úmida). 


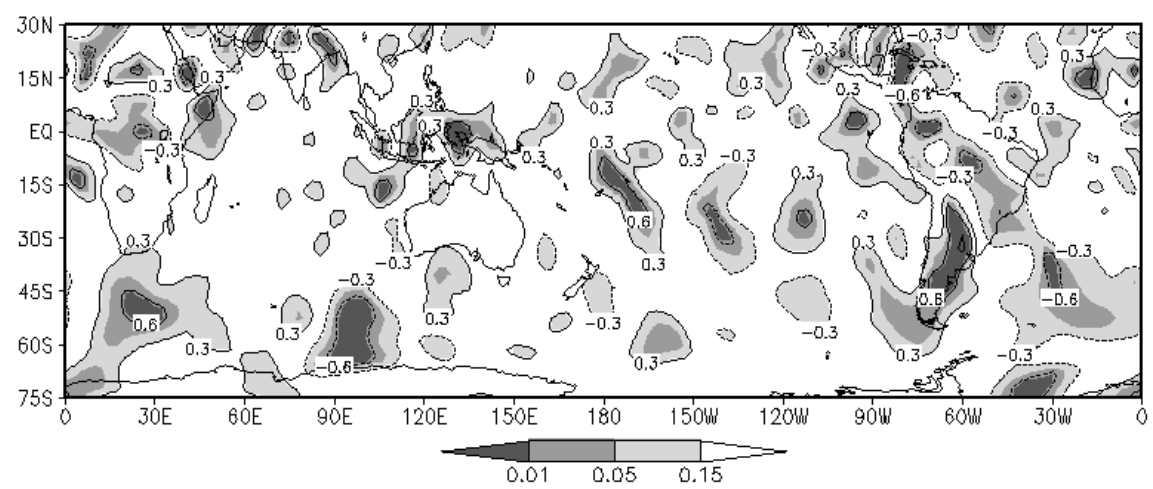

Figura 6 - Correlação entre o vento meridional em $850 \mathrm{hPa}$ e o vento meridional médio em $850 \mathrm{hPa}$ na região de $27^{\circ} \mathrm{S}-40^{\circ} \mathrm{S}$ e $54^{\circ} \mathrm{W}-57^{\circ} \mathrm{W}(\mathrm{Pampa}$ Úmida).

$150^{\circ} \mathrm{W}$ até a costa da América do Sul, embora o padrão do trem de ondas seja muito similar. Em particular, ficam evidentes os núcleos ao sul e ao norte da região de análise. Fisicamente interpreta-se este resultado como a presença da crista ao sul e um cavado ao norte, que são característicos de episódios de GG, conforme mostra as análises de Müller et al. (2005) e estão associadas à diminuição de temperatura observada em superfície.

Estes resultados concordam com as simulações de anomalia de vento em $850 \mathrm{hPa}$, que apresentam um vento de sul afetando principalmente o cone sul do continente (não mostrado) e que se refletem nas correlações positivas significativas $(0,6)$ apresentadas na figura 6 . Este padrão de circulação gera condições favoráveis a ocorrência de GG, uma vez que o ar frio e seco é canalizado ao leste dos Andes, atingindo a região da Pampa Úmida.

\section{CONCLUSÕES}

Através de um experimento numérico utilizando um modelo baroclínico, pôde-se obter um padrão de propagação de onda que atinge o cone sul da América do Sul, e modifica a circulação local, criando condições favoráveis a eventos de geadas generalizadas em certas regiões. As ondas de Rossby são geradas a partir de uma forçante térmica, cuja posição escolhida foi baseada na observação da anomalia de convecção mais significativa obtida através das composições dos dados que contém os invernos de máxima freqüência de ocorrência de GG na região da Pampa Úmeda (PU) na Argentina. O aquecimento diferencial observado na região do Pacifico tropical ocidental a noroeste da Austrália, atuaria como gerador das ondas de Rossby, que se propagam até o continente Sul Americano, favorecendo assim a ocorrência destes eventos. Esse trem de ondas está caracterizado por um número de onda 3 e é semelhante aos resultados apresentados em Müller e Ambrizzi (2006).

O padrão de ondas simulado está bem representado por valores significativos de correlação entre o vento meridional em altos e baixos níveis e a mesma variável na região da PU, assim como, com a temperatura em superfície nesta região. Em particular, os ventos de sul, a leste dos Andes, que são responsáveis por trazer ar frio e seco para a região de interesse foram bem correlacionados.

Estas análises complementam os trabalhos anteriormente apresentados sobre a importância de forçantes remotas em gerar ondas planetárias, que em condições especiais do fluxo básico podem criar as condições necessárias para favorecer eventos de GG sobre a parte centro norte da Argentina.

\section{AGRADECIMENTOS}

Este trabalho foi parcialmente financiado pelo Projecto PIP-114-200801-00591 del Consejo Nacional de Investigaciones Científicas y Técnicas (CONICET), Argentina. Conta-se também com a colaboração das agências brasileiras CNPq, FAPESP e CAPES através dos co-autores do trabalho.

\section{REFERÊNCIAS BIBLIOGRÁFICAS}

Ambrizzi T., Hoskins B.J.; Hsu H. H. Rossby wave propagation and teleconnection patterns in the austral winter. J. Atmos. Sci., 52, 3661-3672, 1995.

Ambrizzi T., Hoskins B.J. Stationary Rossby wave propagation in a baroclinic atmosphere. Quart. J. Meteor. Soc., 123, 919-928, 1997.

Berbery E.H., Nogues-Paegle J. Intraseasonal interactions between the tropics and extratropics in the Southern Hemisphere. J. Atmos. Sci., 50, 1950-1965, 1993.

Cavalcanti I.F.A. Teleconnection patterns orographically induced in model results and from observational data in the austral winter of the Southern Hemisphere. Int. J. Climatol., 20,1191-1206, 2000.

Hoskins B.J,, Simmons A.J. A multi-layer spectral model and the semi-implicit method. 
Quart. J. Meteor. Soc., 101, 637-655, 1975.

Hoskins B.J., Karoly D.J. The steady linear response of a spherical atmosphere to thermal and orographic forcing. J. Atmos. Sci., 38, 1179-1196, 1981.

Hoskins B.J., Jin F.F. The initial value problem for tropical perturbations to a baroclinic atmosphere. Quart.J.Roy. Meteor.Soc., 117, 299-317,1991.

Jin F.F., Hoskins B.J. The direct response to tropical heating in a baroclinic atmosphere. J. Atmos. Sci., 52, 307-319, 1995.

Kalnay E, et al. The NCEP/NCAR 40-year reanalysis project. Bull. Amer. Meteorol. Soc, 1996.

Magaña V., Yanai M. Mixed Rossby-Gravity Waves Triggered by Lateral Forcing. J. Atmos. Sci., 52, Issue 9, 1473-1486, 1995.

Magaña V., Ambrizzi T. Dynamics of subtropical vertical motions over the Americas during El Niño boreal winters. Atmósfera, 18, 4, 2005.

Marengo J.A., Ambrizzi T., Kiladis G e Liebmann B. Upperair wave trains over the Pacific Ocean and wintertime cold surges in tropical-subtropical South America leading to Freezes in Southern and Southeastern Brazil. Theor. Appl. Climat., 74, 243-247, 2002.

Mo K.C., Higgins R.W. The Pacific-South American modes and tropical convection during the Southern Hemisphere Winter. Mon. Wea. Rev., 126, 1581-1596, 1998.

Müller G.V., Nuñez M.N., Seluchi M. Relationship Between ENSO Cycles and Frost Events Within the Pampa Humeda Region. Int. J. Climatol., 20, 1619-1637, 2000.

MüllerG.V.,Ambrizzi T., NuñezM.MeanAtmospheric Circulation Leading to Generalized Frosts in Central Southern South America. Theor. Appl. Climat., 82, No 1-2, 95-112, 2005. Müller G.V., Ambrizzi T. Rossby Wave Propagation and its
Relationship with Severe Frosts over South America. Clivar Exchanges. 11, 3, 18-20, 2006.

Müller G.V. Variabilidad Interanual de las Heladas en la Pampa Húmeda. Rev. Bras. Meteor., 21, No. 1, 135-141, 2006.

Müller G.V., Ambrizzi T. Teleconnection Patterns and Rossby Wave Propagation Associated to Generalized Frosts over Southern South America. Clim. Dyn., 29, No. 6, 633-645, 2007.

Nogues-Paegle J., Mo K. Transient response of the Southern Hemisphere subtropical jet to tropical forcing. J. Atmos. Sci., 45, 1493-1508, 1988.

Sardeshmukh P.D., Hoskins B.J. The generation of global rotational flow by steady idealized tropical divergence. J. Atmos. Sci., 45, 1228-1251, 1988.

Simmons A. J., Burridge D. M. An energy and angularmomentum conserving vertical finite difference scheme and hybrid vertical coordinates. Mon. Wea. Rev., 109, 758-766, 1981.

Ting M., Sardeshmukh P. D. Factors determining the extratropical response to equatorial diabatic heating anomalies. J. Atmos. Sci., 50, 907-918, 1993.

Valdes P.J., Hoskins B.J. Linear stationary wave simulations of the time-mean climatological flow. J. Atmos. Sci., 46, 2509-2527, 1989.

Wilks D.S. Statistical methods in the atmospheric sciences. Academic Press, 1995, 465 pp.

WMO N¹34. Guide to Agricultural Meteorological Practices. 1981. 